\title{
¿El poder para qué?
}

\section{Las relaciones de poder en ambientes virtuales de aprendizaje}

\author{
HERNÁN DARÍO CADENA \\ Poilitécnico Grancolombiano \\ hdcadena@poligran.edu.co
}

Comunicador Social-Periodista de la Universidad de la Sabana; cursa maestría en Informática Educativa en la misma institución. En la actualidad trabaja como profesor de tiempo completo del Departamento de Comunicación del Politécnico Grancolombiano. Ha dedicado la mayor parte de su vida a la docencia, desempeñándose en las universidades Católica de Manizales, Politécnico Grancolombiano y Sabana. Tambén ha colaborado con el área de closedcaption para programas de televisión.

\section{Resumen}

En este documento que reflexiona sobre la evaluación en los nuevos escenarios de aprendizaje virtual, el autor se cuestiona sobre las relaciones de poder generadas por el ejercicio evaluativo y trata de indagar - en un ejercicio exploratorio inicialla manera en que estas se han transformado o no, a partir de las modificaciones sustanciales, otras veces sutiles, del ámbito educativo y de sus principales actores: profesores y estudiantes, tutores y aprendices. De 1gual modo, el texto se ocupa de establecer fronteras y diferencias entre evaluar y calificar, especialmente desde la perspectiva de la educación en los ambientes virtuales de aprendizaje. Asimismo, se interroga sobre la existencia de horizontalidad en los nuevos papeles educativos, a partir de la evaluación, y finalmente, sugiere cómo esta debería ser una herramienta efectiva de gestión del aprendizaje.

\section{Palabras Claves}

Ambiente virtual de aprendizaje, evaluación, relaciones de poder.

\section{Abstract}

This paper reflects on the assessment in the new virtual learning scenarios. The author questions the relations of power generated by the evaluative exercise and examines, in an initial exploratory exercise, the way they have been transformed or not, after substantial changes, sometimes subtle, the field of education and its main actors: teachers and students, tutors and learners. Similarly, the text deals with setting boundaries and differences between assessment and grading, especially from the perspective of education in virtual learning environments. It also questions the existence of horizontality in the new educational roles, from assessment, and finally suggests how this should be an effective tool for learning management.

\section{Key words}

Virtual learning environment, evaluation, power relations.
Fecha de recepción: 10 de septiembre de 2010 Fecha de aprobación: 30 de septiembre de 2010

\section{Introducción}

Tradicionalmente la evaluación se ha considerado una herramienta que permite comprobar cómo está funcionando determinado proceso educativo, tanto por parte del docente como por parte del estudiante; pero, es este último quien ha resultado más "evaluado", por estimarse que es él el sujeto de enseñanza y aprendizaje.

También desde un enfoque clásico, la evaluación se asume como un instrumento para medir: tasa el rendimiento de un estudiante según el rasero del profesor, quien ha impartido un conocimiento para que el alumno lo copie, memorice la mayor parte de él, lo reproduzca y reciba en contraprestación un número por esa labor.

En el mismo sentido, cabe reconocer que la evaluación se convirtió así en un mecanismo de control, de presión-algunas veces de opresión-, que en determinadas circunstancias se erigía como el "arma" de los maestros en la "lucha" contra sus estudiantes.

Desde estas perspectivas es fácil llegar a la inferencia de que la evaluación generaba -y sigue generando- relaciones de poder casi verticales, entre un superior y sus subordinados (profesor-estudiantes), y, por supuesto, esto engendraba -y lo sigue haciendo- un sinnúmero de tensiones entre ambas partes, por lo general resueltas bien sea positiva (cuando el estudiante "pasa"), o negativamente (cuando "se queda"); pero, finalmente, ¿quién se preguntaba por el aprendizaje real ocurrido o cuál había sido el papel del profesor en este proceso? Quizá el sistema educativo bebió mucho de esta fuente y dejó atrofiado en buena parte su sistema hepático: es decir, el encargado de regular, drenar y limpiar. 
Ahora bien, ¿qué pasa con la evaluación en nuevos escenarios educativos? Con la intervención de las tecnologías y la amplia difusión de plataformas y aulas virtuales de aprendizaje, los modos de hacer la educación se han transformado, pero ¿habrá ocurrido lo mismo con la evaluación?

De este modo, la pregunta orientadora del presente artículo reflexivo -que no pretende agotar su respuesta, sino ofrecer algunos indicios preliminares y dejar abierto el espacio para una profundización posterior- es si ¿existen nuevas relaciones de poder, generadas por la evaluación, en un ambiente virtual de aprendizaje?

\section{Saquen una hojita...}

Una frase de este tenor genera algo de zozobra en el ambiente de un salón de clases, nerviosismo, inconformidad y resignación. Es el momento en que el profesor reafirma su posición dominante y los estudiantes asumen la postura de sumisos subalternos. ¿Cuántos de los docentes habrán hecho este tipo de "evaluaciones" sorpresa, tan solo como una manera de controlar al grupo, como esgrimiendo una herramienta de poder? ¿Cuánto habrá incidido esta clase de prácticas -de por sí tan extendidas- en la percepción negativa de los estudiantes hacia la evaluación?

Lo anterior lleva a considerar que la educación tradicional, con un alto componente de presencialidad, puede ser un terreno propicio para que se generen las tensiones entre quien evalúa y quien es evaluado; en todo caso hay una figura visible, al frente del aula, que tiene en sus manos un listado y del cual se espera que imparta contenidos para luego "tomar la lección" y verificar que estos "conocimientos" han sido absorbidos por los alumnos.

En un interesante texto sobre la actividad de evaluar como mecanismo de tener, poder y valer, Pardo et ál (2006, p.4), afirma:

$\mathbb{E}$ poder implica en educación una relación de autoridad entre el maestro y el alumno diferente a otras relaciones. Si consideramos la evalluación al interior de la institución educativa, a partir de la categoría del poder, podríamos asemejar la confrontación de esta acción con un retén donde la autoridlad ejerce control sobre el sujeto: el alumno debe obedecer y parar cuando la autoridad (iinstitución o maestro) se lo mande, es decir, en aquellos momentos especiales de evaluaciones trimestrales, finales, etc., para que pueda ser examinado y controlado por una persona idónea y competente que está facultada para dar órdenes y dar luz verde para seguir una buena nota; o luz roja para retenerlo en un lugar: la nota rajada, la mala nota, escrita en rojo, de manera que haga acopio de información que le permita re- portar que se está "bajo control o fuera de control", es decir, al margen de la ley o dentro de los parámetros normales.

El hecho de que exista un evaluador, con la potestad para determinar si lo que un estudiante hace está bien o mal, sin duda genera unas relaciones de poder subyacentes en el proceso educativo. Y, probablemente, sea uno de los contratos que con más claridad pueda ser entendido entre los actores de este proceso, a pesar de cualquier rótulo que se le imponga o cualquier empaque en el que se entregue la evaluación.

\section{Evaluación vs. calificación}

Ahora bien, quizás desde la propia naturaleza del hombre hay una tendencia a juzgar lo que ocurre en el medio ambiente, sean actitudes, comportamientos o acciones de los otros. La educación -entre otras actividades humanas- formalizó ese juzgar y lo llamó "evaluación", pero encauzándolo con un noble propósito: ayudar al mejoramiento de quienes se están educando.

Sin embargo, cuando dicha práctica empezó a incluir números, cifras para "medir" la cantidad de aprendizaje de un estudiante, quizás allí se dio un punto de quiebre entre lo que en realidad significa evaluar y el acto de calificar. La segunda empezó a reemplazar a la primera de manera ímplicita.

Las escalas de 1 a 10, de 0 a 5, de A a F, o de E a I, aunque si bien es cierto permitieron una organización administrativa de la información evaluativa, también empezaron a crear escenarios propicios para los intercambios entre conocimientos y notas, y tácitamente permitieron que la evaluación se convirtiera en asunto de un solo momento y no en una herramienta del proceso de mejoramiento de los estudiantes: estudiar para el examen, ¿suena familiar?

Por su parte, desde otros enfoques de la educación, y más aquellos que pretenden integrar las Tic (técnicas de información y comunicación), los ambientes virtuales, las comunidades de estudio, se ha venido considerando la evaluación como una prioridad; como un elemento crucial dentro del proceso educativo más que una molestia. Al respecto, dice Campaña (2009, p.2):

Evaluar en el aprendizaje significa analizar y reflexionar sobre la enseñanza que estamos impartiendo para luego tomar decisiones que repercutirán en el futuro. Es decir, adecuar nuestras prácticas docentes a lo que, querámoslo o no, necesita el estudiante. Esto implica recurrir a estrategias que nos entreguen información lo más real posible respecto a lo que se ha logrado, avances, errores y dificulltades de los estudiantes ante un contenido específico, en- 
tre otros. Luego, para fines administrativos, esa evaluación se debe traducir en una calificación o cualificación seguín sea el caso.

Allí, claramente, se ve involucrada la calificación, pero como parte de un proceso mucho más amplio que es el de evaluar. Es importante tener esta claridad, en especial a la hora de abordar la evaluación desde los ambientes virtuales de aprendizaje.

De manera adicional, tratar este tema expone un abanico de preguntas que merecen un desarrollo posterior en espacios de investigación más profundos: ¿se trata de "transferir" los modelos tradicionales de evaluación a este tipo de ambientes, o realmente se genera un nuevo tipo de evaluación?, ¿la evaluación en estos ambientes "se toma en serio"?, ¿los tutores utilizan la evaluación para "alinear" a los participantes?, ¿hay o no claridad sobre los modelos de evaluación que se deben utilizar en estos escenarios?, ¿el hecho de que en estos ambientes de aprendizaje haya horizontalidad altera la relación tradicional de poder entre profesor-alumno?, ¿existe realmente la horizontalidad o esta se rompe en el momento de evaluar y de nuevo hay verticalidad?

\section{¿Yentonces?}

Es claro que en la actualidad hay un marcado interés por privilegiar el aprendizaje autónomo -casi en contraposición a la enseñanza- y a considerar aprendices tanto a los estudiantes como a los docentes. En este contexto, los métodos tradicionales necesitan, entonces, nuevas miradas. Así lo propone Martínez (2009, p.1):

La forma tradicional de evaluación no hace justicia a las consideraciones teóricas, epistemológicas y pragmáticas de la educación a distancia. Mucho menos a las posibilidades de desarrollo de competencias donde el fundamento esencial es la posibilidad de mejoramiento permanente en la adquusisición y desarrollo de las mismas. Esto signica que necesariamente se deba plantear un paradigma para este proceso, que supere la rendición de cuentas sobre la enseñanza, coarte la toma libre y responsable de decisiones, desconozca el interés y necesidades del estudiante, la construcción colectiva del conocimiento así éste ya esté formalmente dado como ocurre en los procesos de aprendizaje y se supere la discordia estudiante-docente ante la necesidad de obtener la nota aprobatoria sin que afecte demasiado la estructura cognitiva de los actores del proceso de enseñanza-aprendizaje

Comprendido lo anterior, lo que se puede intuir acerca de los ambientes virtuales de aprendizaje es que así como hay una transición de la figura del profesor al tu- tor, y del estudiante al aprendiz, además de las necesarias modificaciones en contenidos, nuevas reflexiones pedagógicas y trabajo con herramientas TIC, la evaluación supone un cambio profundo que aún está en proceso de desarrollo y que se constituye en un verdadero reto para los educadores, tal como lo expone el mismo Martínez más adelante (2009, p.1):

La evaluación en la educación a distancia se concibe y ejecuta como un proceso inserto en la construcción del conocimiento basado en la acción y la reflexión que sobrepase la repetición para llegar al conocimiento y de la memoria al razonamiento. Así, ayuda a la generación de mültiples oportunidades para que el estudiante piense, cuestione, reelabore el conocimiento y lo exprese de muchas maneras. Se caracteriza por ser un ejercicio de racionalidad comunicativa basada en la valoración de los juicios que en igualdad de condiciones expresen los actores del proceso buscando sul entendimiento y proponiendo acciones de mejoramiento, que superen la subjetividad que siempre señala un proceso evaluativo [ $\cdots$.

Es de notar que allí se menciona un ejercicio de racionalidad en la que los actores expresan los juicios en igualdad de condiciones. Por tanto, desde esta perspectiva e idealmente, la evaluación en ambientes virtuales de aprendizaje, como puede serlo la utilizada en la educación a distancia, implica una afirmación de la horizontalidad y democratización del proceso evaluativo; esto fácilmente llevaría a pensar que las relaciones de poder de las que se hablaba al inicio de este artículo tienden a disolverse, pues también hay un cambio de papeles implícito en los nuevos escenarios educativos, en los que las estructuras jerárquicas funcionan de otras maneras. Sin embargo, aún hay un amplio terreno por explorar para convalidar en la práctica qué tan cierto es esto.

Evidentemente, las preguntas siguen abiertas y la evaluación en los ambientes virtuales merece una larga reflexión, mucho más en estas latitudes en las que se importa constantemente lo que pasa en otras realidades. Los nuevos actores de los procesos educativos mediados por tecnologías, tanto docentes como estudiantes, deberían ver en la evaluación una completa y versátil herramienta para gestionar efectivamente su proceso de aprendizaje, y no un arma contundente con la cual determinar quién tiene el poder. 


\section{Referencias}

1. Campaña, K. (2009). Evaluación de los aprendizajes, nuestra gran y constante preocupación. Obtenida el 30 de noviembre de 2009, de http://www.quadernsdigitals. net/index.php?accionMenu=hemeroteca.VisualizaArticulolU.visualiza\&articulo_id=10892

2. Martínez, A. (2009). Evaluación en los ambientes virtuales de aprendizaje. Evaluación por competencias. Obtenia el 26 de noviembre de 2009, de http://cnx.org/content/ m16546/1.2/

3. Pardo, D., Bejarano, B., Henao, M., y Valero, M. (2006). Evaluar: tener, poder y valer. Revista Iberoamericana de Educación, (40) 2006. OEl.

\section{Bibliografía}

1. Bates, A. (1995). Technology, Open Learning and Distance Education. Nueva York: Rotledge.

2. Chaupart, J. (1998). El tutor, el estudiante y su nuevo rol. Desarrollo de ambientes de aprendizaje en educación a distancia. (p. 97-110). Universidad de Guadalajara.

3. Dávila, S. (2010, enero). El aprendizaje significativo. Esa extraña expresión. Contexto Educativo. Consultada el 23 de enero de 2010, http://contexto-educativo.com.ar

4. Duart, J. (2002). Evaluación de la calidad docente en entornos virtuales de aprendizaje. Barcelona: Universitat Oberta de Catalunya (UOC).

5. Henao, O. (2002). La enseñanza virtual en la educación superior (1 a. ed.). Colombia: Icfes.

6. Loughling, C. (1996). El ambiente de aprendizaje: diseño y organización. España: Morata.

7. Pérez, A. (2009). La evaluación en un entorno virtual. Mérida (Venezuela): Academia, Universidad de los Andes, año 6(11).

8. Pisanty, A. (2000). Dos taxonomías de los medios técnicos para la educación a distancia. Revista Digita Universitaria. México: Unam, 1(1).

9. Sangrà, A. (2002). La calidad en las experiencias virtuales de educación superior. Barcelona: Universitat Oberta de Catalunya (UOC).

10. Silvio, J. (2006). Hacia una educación virtual de calidad, pero con equidad y pertinencia. Revista de Universidad y Sociedad del Conocimiento. Barcelona: Universitat Oberta de Catalunya (UOC), 3(1).

11. Silvio, J. (2010). Reflexiones sobre la calidad en la educación virtual. Obtenida el 10 de enero de 2010, de http:// www.educoas.org/portal/bdigital/lae-ducacion/139/ pdfs/139pdf4.pdf
12. Willging, P. (2008). Técnicas para el análisis y visualización de interacciones en ambientes virtuales. Revista Hispana para el Análisis de Redes Sociales, Barcelona: Universitat Autònoma de Barcelona, (14). 